Check for updates

Cite this: Phys. Chem. Chem. Phys., 2022, 24, 1456

Received 5th November 2021 Accepted 18th December 2021

DOI: $10.1039 / \mathrm{d} 1 \mathrm{cp} 05068 \mathrm{k}$

rsc.li/pccp

\section{Discerning phase-matrices for individual nitride inclusions within ultra-high-strength steel: experiment driven DFT investigation $\dagger$}

\author{
Andrey A. Kistanov, (D) *ab Ekta Rani, (D) ${ }^{a}$ Harishchandra Singh, (D) *ab Timo Fabritius, ${ }^{\text {bc }}$ \\ Marko Huttula iD ab and Wei Cao iD ab
}

\begin{abstract}
Non-metallic inclusions play a decisive role in the steel's performance. Therefore, their determination and control over their formation are crucial to engineer ultra-high-strength steel. Currently, bare experimental approaches are limited in the identification of non-metallic inclusions within microstructural phases of complex steel matrices. Herein, we performed a density functional theory study on the characteristics of different nitride inclusions as observed in spectro-microscopy studies. As per the simulations, TiN inclusions preferentially formed in the austenite matrix, while the ferrite matrix generally hosts $\mathrm{BN}$ inclusions. Furthermore, although the presence of both $\mathrm{BN}$ and $\mathrm{TiN}$ inclusions in the $\mathrm{Fe}_{3} \mathrm{C}$ matrix is possible, their formation is impeded because of the strong inclusion-carbon interactions. The observed regularity in the formation of nitride inclusions in different phases of steel was also confirmed by the comparison of simulated and experimental K-edge XAS spectrum of nitride inclusions. Our work shed the light on the formation of nitride inclusions in different steel matrices and facilitates their further experimental identification.
\end{abstract}

\section{Introduction}

Owing to a low-cost production along with a wide range of strength and ductility properties, ferrite-martensite dual-phase ultra-high-strength steel is highly desirable for automotive, civil engineering, and automobile manufacturing applications. ${ }^{1-3}$ However, during steel manufacturing, the formation of nonmetallic inclusions (NMIs) within multi-phase steel, typically in low or very low volume fractions, is unavoidable. NMIs are regions of non-metallic chemical compounds, such as oxides, sulfides, and nitrides, which are formed during the different stages of the steel production. An in-depth understanding of NMIs, including their composition, size, shape, and amount, is an imperative step in steel production as they can create serious problems ${ }^{4}$ such as breakage of steel wires during drawing, hydrogen-induced cracking, fatigue failure, and surface flaws. For example, in Al-killed steels, the AlN inclusion can precipitate and induce an intergranular fracture. ${ }^{5}$ The Ti-based larger TiN inclusion, on the other hand, can deteriorate material toughness by promoting cleavage crack propagation. ${ }^{6}$ However,

\footnotetext{
${ }^{a}$ Nano and Molecular Systems Research Unit, University of Oulu, 90014 Oulu, Finland. E-mail: Andrey.Kistanov@oulu.fi, Harishchandra.Singh@oulu.fi

${ }^{b}$ Centre for Advanced Steels Research, University of Oulu, 90014 Oulu, Finland

${ }^{c}$ Process Metallurgy Research Unit, University of Oulu, 90014 Oulu, Finland

$\dagger$ Electronic supplementary information (ESI) available. See DOI: 10.1039/ d1cp05068k
}

the formation of $\mathrm{BN}$ inclusions is not encouraged as it can hinder the role of boron, used as a microalloying element in high-strength steels to improve hardenability. ${ }^{7}$

Spectro-microscopy studies on NMIs embedded in steel ${ }^{8}$ indicated that additional $\mathrm{B}$ atoms result in h-BN along with a strong interaction with a Ca-based inclusion. These Ca-based inclusions also stabilize with TiN. However, no interaction between TiN and $\mathrm{BN}$ within the studied steel matrix was found, and the reason behind the same could not be deciphered. Thus, although nitride inclusions in the steel matrix are observed, it is difficult to define their stability and to probe which phase they are in due to the limitations of available experimental methods for such determinations. In such experimental limitations due to the complex microstructure of steel matrix and hidden inclusions within, different computational approaches including the atomic multiplet theory, ${ }^{9,10}$ delta-self-consistent splitting and the Bethe-Salpeter equation methods ${ }^{11}$ have been proposed to realize the physical mechanism of inclusion formation in different systems including a few variants of steel. For instance, these approaches can be successfully utilized for the investigation of shape and morphology of inclusions in metallic materials, ${ }^{12}$ prediction of the effect of inclusions on the hydrogenation of steels, ${ }^{13}$ and differentiation of elements via their X-ray absorption (XAS) spectral simulations. ${ }^{14}$ Despite the progress described above, investigating nitride inclusions within multi-phase carbon steel is still posing challenges.

Herein, we conducted a density functional theory (DFT)-based study, supplemented by experimental spectro-microscopic data, 
on the formation of nitride inclusions in different phases of steel together with simulated spectroscopic evidence, i.e., K-edge XAS spectrum. In this study, the discovered regularity in the formation of specific inclusions depending on the type of matrix can further shed light on the controlled formation of nitride inclusions in a particular phase in commercial steel production.

\section{Methods}

The computational part was carried out in the framework of DFT as implemented in the Vienna $\mathrm{Ab}$ initio Simulation Package (VASP) code. ${ }^{15}$ To simulate NMIs in the steel matrix, the supercell method was used. The geometry optimization of iron matrices (created based on the data in ref. 16) and inclusion-containing iron matrices was treated based on the Perdew-Burke-Ernzerhof functional within the generalizedgradient approximation. ${ }^{17}$ Bulk crystals of bare austenite, ferrite, and $\mathrm{Fe}_{3} \mathrm{C}$ were fully optimized. The optimization process was stopped when the atomic forces and total energy values were smaller than $0.01 \mathrm{eV} \AA^{-1}$ and $10^{-6} \mathrm{eV}$, respectively. A kinetic energy cutoff of $520 \mathrm{eV}$ was adopted. The Brillouin zone was sampled with a $3 \times 3 \times 3 k$-mesh grid for the structure optimization step. The obtained lattice parameters of austenite were $a=b=c=3.646 \AA$, those of ferrite were $a=b=c=2.839 \AA$, and those of $\mathrm{Fe}_{3} \mathrm{C}$ were $a=4.491 \AA, b=5.030 \AA, c=6.739 \AA$, which agreed well with previous theoretical and experimental results. ${ }^{18,19}$ For the calculation of the XAS spectrum, the supercell core-hole method as implemented in the VASP code was used. ${ }^{16}$ For the XAS spectrum calculations, the inclusioncontaining structures were created in several steps (Fig. 1). At the first step, supercells (either austenite, ferrite or $\mathrm{Fe}_{3} \mathrm{C}$ ) were created based on the previously optimized unitcells of crystals. Particularly, a $3 \times 3 \times 3$ supercell (172 iron atoms) of the unitcells of austenite was used. A ferrite supercell model consisting of 122 iron atoms was created of $4 \times 4 \times 4$ unitcells. $\mathrm{A} \mathrm{Fe}_{3} \mathrm{C}$ supercell model consisting of 144 iron atoms and 48 carbon atoms was created of $3 \times 2 \times 2$ unitcells. At the second step, cluster-like inclusions (BN, TiN, and AlN)

1. Steel matrix model

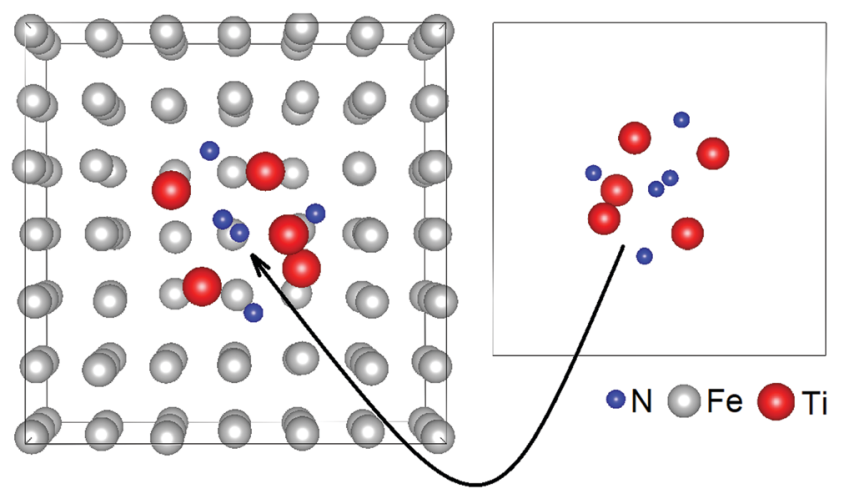

3. Insert cluster

Fig. 1 Schematic representation of the inclusion-containing steel matrix model preparation. consisting of 10 atoms were created and preoptimized. At the third step, the atoms located in the middle of steel matrixes were removed, and the cluster-like inclusions were placed instead. The final structures, which were inclusion-containing steel matrices, were optimized. Periodic boundary conditions were applied in all three transverse directions. To avoid the core-hole effect from the neighboring cells, the considerably large supercells with a size not smaller than $11 \times 11 \times 11 \AA$ were considered. The structures were fully optimized until the atomic forces and total energy values were smaller than $0.01 \mathrm{eV} \AA^{-1}$ and $10^{-6} \mathrm{eV}$, respectively. A kinetic energy cutoff of $520 \mathrm{eV}$ was adopted. A $9 \times 9 \times 9 \mathrm{k}$-mesh grid in the Brillouin zone was used. The supercell core-hole based on the representation of a solid as a supercell using periodic boundary conditions and a core hole was created by removing a core electron and placing it into the conduction bands.

Formation energy $E_{\text {form }}$ of bare and inclusion-containing steel matrices was defined as

$E_{\text {form }}$

$$
=\frac{E_{\mathrm{tot}}-N_{\mathrm{Fe}} E_{\mathrm{Fe}}-N_{\mathrm{N}} E_{\mathrm{N}}-N_{\mathrm{B}} E_{\mathrm{B}}-N_{\mathrm{Al}} E_{\mathrm{Al}}-N_{\mathrm{Ti}} E_{\mathrm{Ti}}-N_{\mathrm{C}} E_{\mathrm{C}}}{N_{\mathrm{Fe}}+N_{\mathrm{N}}+N_{\mathrm{B}}+N_{\mathrm{Al}}+N_{\mathrm{Ti}}+N_{\mathrm{C}}}
$$

where $E_{\mathrm{tot}}$ is the total energy of the system, and $E_{\mathrm{Fe}}, E_{\mathrm{N}}, E_{\mathrm{B}}, E_{\mathrm{Al}}$, $E_{\mathrm{Ti}}$, and $E_{\mathrm{C}}$ correspond to the total energy per atom (the energy of a single atom in the bulk structure) of $\mathrm{Fe}, \mathrm{N}, \mathrm{B}, \mathrm{Al}, \mathrm{Ti}$, and $\mathrm{C}$ elements, respectively. The values of the formation energies of bare matrixes well matched with the reference data. ${ }^{18}$

The sample of interest for this investigation was a commercial low-alloyed carbon steel with a nominal composition of $0.15 \mathrm{C}, 0.3 \mathrm{Si}, 1.0 \mathrm{Mn}, 0.4 \mathrm{Cr}$ in $\mathrm{wt} \%$. Its structural phase, micrograph, and spectro-microscopic measurements were recorded and analyzed via high energy synchrotron X-ray diffraction, FE-SEM and X-PEEM, respectively. Incident beam of a $2 \times 10^{-4}$ bandwidth and photon flux of 1-5 $\times 10^{12} \mathrm{ph} \mathrm{s}^{-1}$ were set. A SX700 monochromator with $\mathrm{Au} / \mathrm{Si}$ 1200-line per mm grating was used to probe the B K-edge, $\mathrm{N}$ K-edge, and Ti L 2,3-edge XAS. The incident photon energy was scanned at a $0.2 \mathrm{eV}$ step for $\mathrm{B}$ K-edge and N K-edge, and a step of $0.1 \mathrm{eV}$ was set for L 2,3-edge. More details of the experimental setup were presented in our previous report. ${ }^{8}$

\section{Results and discussion}

Fig. 2 presents the SEM image of the steel matrix (Fig. 2a) hosting BN and TiN inclusions, computational model of inclusions in steel matrix (Fig. 2b) and enlarged $\mathrm{BN}$ and TiN inclusions (Fig. 2b and c). According to the HE-SXRD data (Fig. 2e), ferrite was the major phase in the studied sample, a small amount of retained austenite and cementite phases can also be in place. ${ }^{8}$ It is known that NMIs of the ppm-level concentration are hardly detectable through HE-SXRD, ${ }^{20}$ and the SEM-EDS mapping analysis only provides the elemental information. However, based on the SEM-EDS mapping analysis, we found that the selected inclusions in the steel sample 


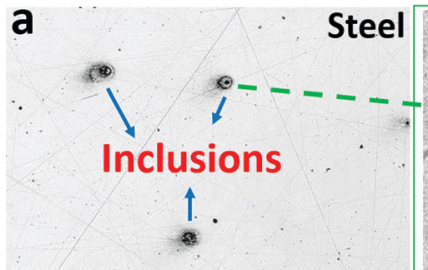

b

were nitrides with mostly $\mathrm{Ti}$ and $\mathrm{B}$ compounds, and the possible presence of Al-based compounds. ${ }^{8}$

To get insights into the stability of different nitride inclusions in common steel matrices, DFT-based calculations were conducted. Three nitride inclusions experimentally observed in the studied sample, BN, TiN, and AlN, were considered in three experimentally observed matrices, which are austenite, ferrite, and $\mathrm{Fe}_{3} \mathrm{C}$. Based on the formation energy, $E_{\text {form }}$ calculations (Table 1), the formation of $\mathrm{BN}$ inclusions was more energetically favourable in the ferrite matrix, while TiN inclusions were more stable in the austenite matrix. Both $\mathrm{BN}$ and TiN inclusions may exist in the $\mathrm{Fe}_{3} \mathrm{C}$ matrix, but their formation energy was considerably high. In all studied matrices the formation of AlN inclusions was found to be less energetically favourable compared to the formation of BN and TiN inclusions. Further, the comparison of calculated and experimental XAS spectra was performed to elucidate the similarities and origins of nitride inclusions in common steel matrices based on spectroscopic features.

Proceeding from the formation energy calculations, XAS analysis was only performed for the TiN-containing austenite, $\mathrm{BN}$-containing ferrite, and $\mathrm{BN}$-containing $\mathrm{Fe}_{3} \mathrm{C}$ matrices. Previously, it has been confirmed that characteristics of XAS spectra for the B K-edge and $\mathrm{N}$ K-edge in h-BN/metal interfaces can be reasonably reproduced by DFT calculations. ${ }^{14}$ Here, experimentally measured XAS spectra for h-BN and TiN inclusions in the steel sample, DFT-calculated XAS spectra for BN and TiN inclusions in different steel matrices (computational models) and DFT-calculated XAS spectra for bulk h-BN and TiN were compared. Such a comparison aimed to validate our calculations and targeted to facilitate the identification of nitrogen-based inclusions within common steel matrixes.

The comparison of experimental and calculated XAS spectra for the $\mathrm{N} \mathrm{K-edge} \mathrm{and} \mathrm{Ti} \mathrm{K-edge} \mathrm{together} \mathrm{with} \mathrm{the} \mathrm{computational}$ model of the TiN inclusion in the austenite matrix is shown in
Table $1 E_{\text {form }}(\mathrm{eV})$ of bare and inclusion-containing steel matrices

\begin{tabular}{lllll}
\hline Steel matrix & Bare & BN-containing & TiN-containing & AlN-containing \\
\hline Austenite & 0.15 & -0.45 & $-\mathbf{0 . 6 2}$ & -0.45 \\
Ferrite & 0.14 & $-\mathbf{0 . 4 1}$ & -0.26 & -0.19 \\
$\mathrm{Fe}_{3} \mathrm{C}$ & 0.01 & $\mathbf{- 0 . 1 3}$ & $\mathbf{- 0 . 1 2}$ & -0.06
\end{tabular}

Fig. 3. According to Fig. 3a, the XAS spectrum for the N K-edge can be divided into two regions. The first region was represented by the doublet at threshold (marked as n1 and n2), and was formed by unoccupied $\mathrm{N} 2 \mathrm{p}$ states, which are hybridized with Ti 3d states. The presented peaks are sharp with the width of each sub-band of $\sim 2.5 \mathrm{eV}$ (experiment, red line) and $\sim 1.5 \mathrm{eV}$ (DFT, green and black lines). The second region corresponds to more broadened bands (marked as n3 and $\mathrm{n} 4$ ), which are unoccupied N 2p states hybridized with Ti 4sp states. In this region, a larger dispersion of bands with a width of $\sim 5 \mathrm{eV}$ was observed in experiment (red line), while DFT calculations (green and black lines) depicted a smaller width of $\sim 2.5-3 \mathrm{eV}$.

A typical K-edge XAS spectrum for Ti involves the main absorption edge corresponding to transitions from the $1 \mathrm{~s}$ to $4 \mathrm{p}$ states and the pre-edge, which covers transitions from the $1 \mathrm{~s}$ to $3 \mathrm{~d}$ states. The pre-edge feature of the Ti K edge was mostly altered by the symmetry effect of the coordination of Ti with its surrounding atoms. ${ }^{21}$ According to Fig. $3 \mathrm{~b}$, the XAS spectrum of the Ti K-edge consists of weak intensity pre-edge peaks and edge peaks that are labeled as $t 1, t 2, t 3$, and $t 4$. Despite the simplicity of the computational model, the calculated XAS spectra of the $\mathrm{N}$ K-edge and Ti K-edge for the TiN inclusion in the austenite matrix well repeated the experimentally obtained one for the TiN inclusion in the steel matrix. Therefore, it can be proposed that austenite was the most probable host matrix for the TiN inclusion in our steel sample. It should be noted that Ti L-edges were not discussed as DFT 


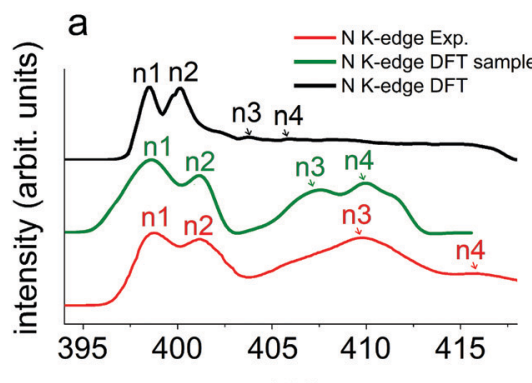

(eV)
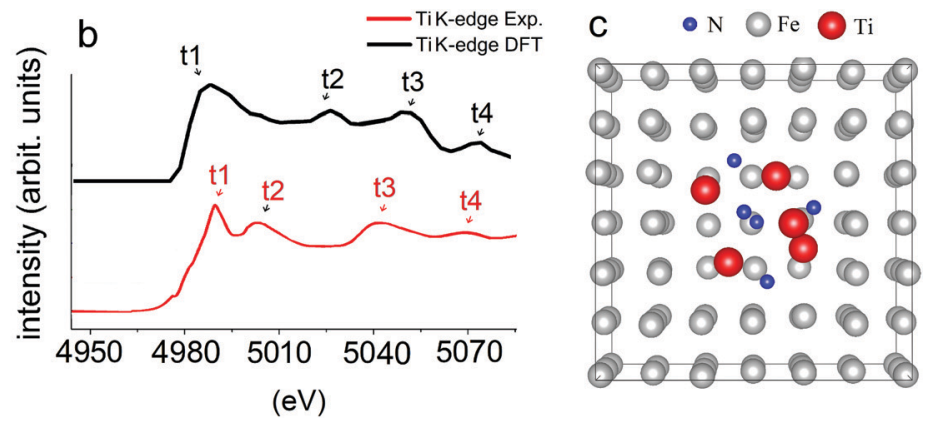

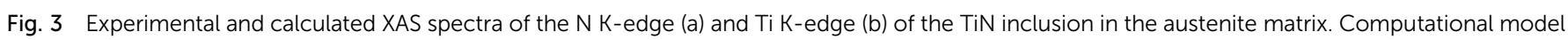
of the TiN inclusion in the austenite matrix (c).

approaches may be unable to adequately describe the L-edges of metals as these edges are dominated by multi-electronexcitation effects. ${ }^{22}$ More detailed discussion is presented in Fig. S1 (ESI $\dagger$ ).

Fig. 4 shows the comparison of experimental and calculated XAS spectra of B K-edge and N K-edge together with the model of the BN inclusion in the ferrite matrix. The broadened $\pi^{*}$ states of boron, visible at the b1 region (Fig. 4a), may not be related to the nature of $\mathrm{B}$ atoms but to the anisotropy of their bonds, which can lead to the incomplete screening of the corehole in this structure. ${ }^{23}$

Bond anisotropy is clearly depicted in Fig. 5 from the asymmetry of XAS spectra of individual $\mathrm{B}$ and $\mathrm{N}$ atoms of the $\mathrm{BN}$ inclusion in the ferrite matrix. Moreover, at the b2 and b3 regions, the localized $\sigma^{*}\left(2 \mathrm{p}_{x}, 2 \mathrm{p}_{y}\right)$ antibonding states between $\mathrm{B}$ and $\mathrm{N}$ atoms were observed on both DFT (DFT, green and black lines) and experimental XAS spectrum plots (red line) in Fig. 4a. Considering that DFT-calculated $\mathrm{N}$ K-edge of the BN inclusion in the ferrite matrix has similar characteristic features as those of experimentally measured ones (Fig. 4b), it can be proposed that ferrite is the host matrix for the TiN inclusion in our sample.

According to Table $1, E_{\text {form }}$ of inclusions in the $\mathrm{Fe}_{3} \mathrm{C}$ matrix is higher than that of inclusions in the austenite and ferrite matrixes. This was also confirmed by XAS analysis; for instance, the spectral shape of the B K-edge (Fig. 6a) and $\mathrm{N}$ K-edge (Fig. 6b) of the $\mathrm{BN}$ inclusion in the $\mathrm{Fe}_{3} \mathrm{C}$ matrix significantly
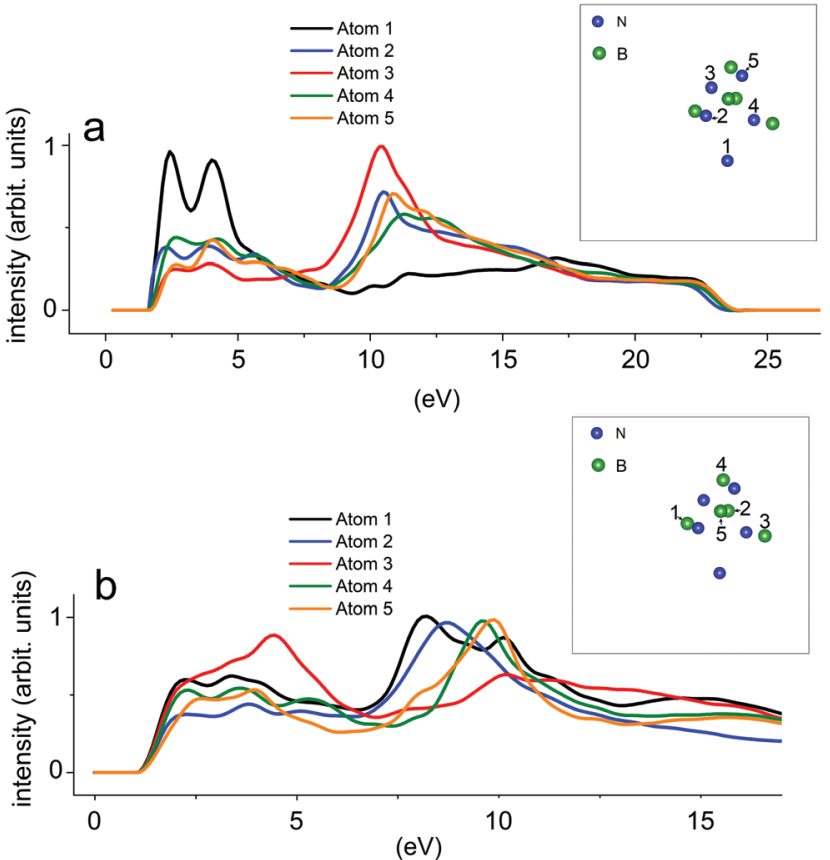

Fig. 5 Calculated XAS spectra of N K-edge (a) and B K-edge (b) for individual atoms of the $\mathrm{BN}$ inclusion in the ferrite matrix. Inset figures show the BN inclusion with the numeration of atoms.

broadened compared to those in other considered matrices. Particularly, the b1 region of the B K-edge was constructed of
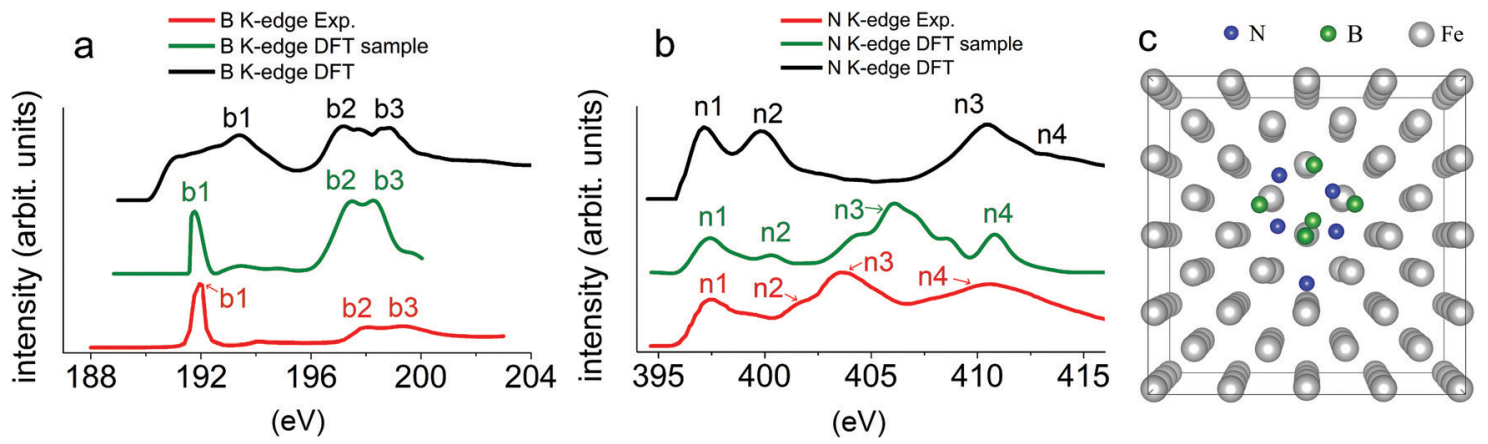

Fig. 4 Experimental and calculated XAS spectra of B K-edge (a) and N K-edge (b) of the BN inclusion in the ferrite matrix. Computational model of the $\mathrm{BN}$ inclusion in the ferrite matrix (c). 
the $\pi$-bonded states of individual B atoms of the BN inclusion. Indeed, the XAS spectra of individual $\mathrm{B}$ and $\mathrm{N}$ atoms of the $\mathrm{BN}$ inclusion (Fig. 7) in the $\mathrm{Fe}_{3} \mathrm{C}$ matrix are significantly asymmetric, and the center of their peaks shifted towards each other, which suggests that the atomic orbitals of the inclusion's atoms were mixed with those of the matrix's atoms. The density of states (DOS) analysis (Fig. S2, ESI $\dagger$ ) of the BN inclusion's atoms in the $\mathrm{Fe}_{3} \mathrm{C}$ matrix reinforced this presumption as a strong hybridization of C-p states with B-p and N-p states was found. A similar behaviour was expected for the TiN inclusion in the $\mathrm{Fe}_{3} \mathrm{C}$ matrix, as shown in the DOS plot in Fig. S3 (ESI $\dagger$ ). Therefore, the inclusions are less stable and significantly disordered in the $\mathrm{Fe}_{3} \mathrm{C}$ matrix compared to the austenite and ferrite matrices.

As mentioned in the introduction, the formation of different nitride inclusions, such as $\mathrm{Al}, \mathrm{BN}$, and TiN, is highly possible in steels. These nitride inclusions play different roles in steel performance. Therefore, the proper characterization of nitride inclusions in steels is required to control their unwanted formation. Based on the stability of the individual nitrides in individual phases of the steel matrix, one can control the formation of NMIs indirectly. This means that during the metallurgy process, just like one can control the phases in the steel matrix and so possibly the associated formation of individual nitride inclusions.

A previous experimental work coupled with DFT-based calculations $^{8}$ investigated the structure, local bonding structure, and electronic properties of several NMIs, and their interaction mechanism within and the steel matrix. SEM-EDS mapping analysis revealed that most inclusions in the steel sample were nitrides with mostly titanium and boron compounds and possible presence of aluminum-based compounds. Although nitride inclusions in the steel matrix were observed, it is difficult to define their stability and to probe which phase they are in due to the complex microstructure of steel and limitations of available experimental methods for such determinations.

In this study, an in-depth investigation of the formation of nitride-based NMIs in common steel matrices was performed via experiment-supported DFT-based calculations. Particularly,
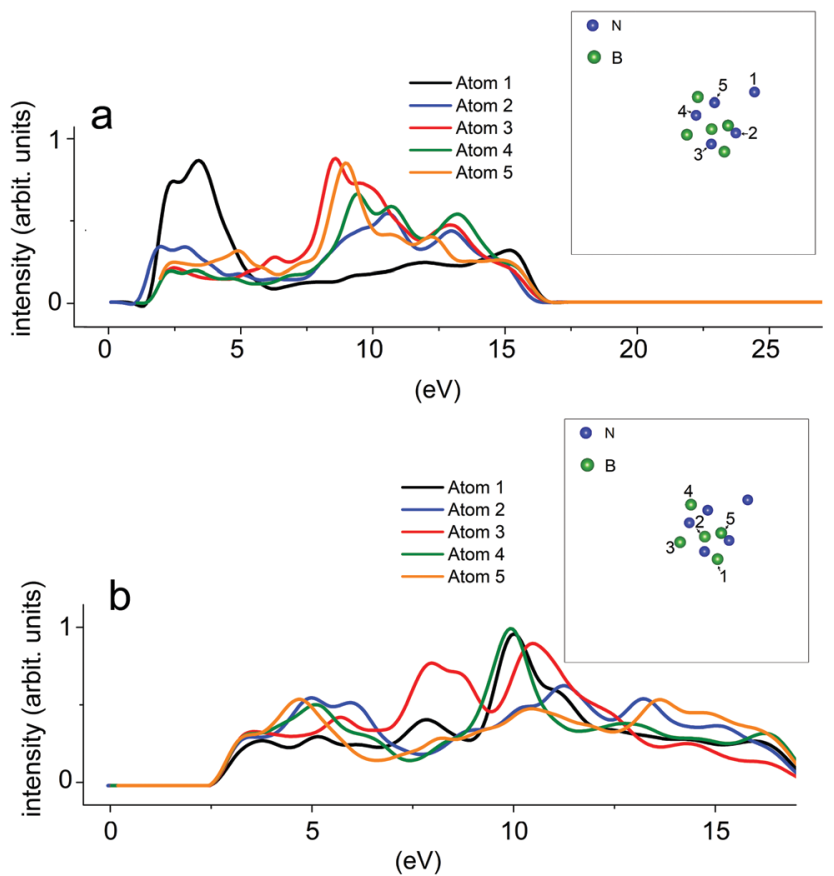

Fig. 7 Calculated XAS spectra of the $\mathrm{N} \mathrm{K}$-edge (a) and B K-edge (b) for individual atoms of the $\mathrm{BN}$ inclusion in the $\mathrm{Fe}_{3} \mathrm{C}$ matrix. Inset figures show the $\mathrm{BN}$ inclusion with the numeration of atoms.

experimental spectromicroscopic data were implemented in a theoretical model describing nitride-based NMIs in common steel matrices. Further, based on this model, DFT-based simulations were utilized to discover the formation of specific nitride-based NMIs depending on the type of the steel matrix based on their formation energies and XAS spectra. Although the occurrence of TiN inclusions in single-phase ferrite ${ }^{24}$ and $\mathrm{BN}$ inclusions in single-phase austenite ${ }^{25}$ is possible and has been noted beforehand, our results suggested a certain preference of each inclusion in the mixed-phase steel. It was found that TiN inclusions preferentially formed in the austenite matrix, while the ferrite matrix generally hosted $\mathrm{BN}$ inclusions. Furthermore, although the presence of both TiN and BN inclusions in the $\mathrm{Fe}_{3} \mathrm{C}$ matrix is possible, their formation was
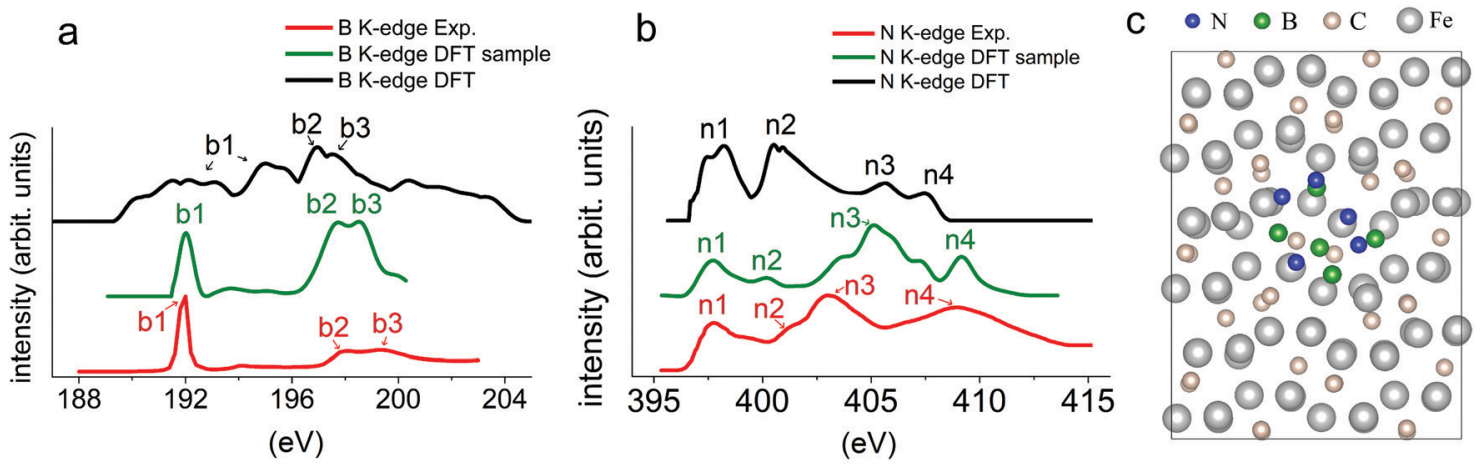

Fig. 6 Experimental and calculated XAS spectra of B K-edge (a) and N K-edge (b) of the BN inclusion in the Fe ${ }_{3} \mathrm{C}$ matrix. Computational model of the BN inclusion in the $\mathrm{Fe}_{3} \mathrm{C}$ matrix (c). 
hampered due to a strong interaction of the inclusions' atoms with the carbon atoms in the matrix.

\section{Conclusions}

Our recent spectro-microscopic investigations demonstrated the formation of $\mathrm{BN}$ and TiN inclusion stabilized with a Ca-based inclusion. ${ }^{8}$ However, BN and TiN inclusions did not co-exist within the same inclusion. In order to decipher the reason behind their hatred nature for each other, we have carried out detailed DFT studies to calculate formation energies and XAS along with DOS, which showed that TiN inclusion preferentially formed in the austenite matrix, while the ferrite matrix generally hosted $\mathrm{BN}$ inclusions. Furthermore, although the presence of both $\mathrm{BN}$ and $\mathrm{TiN}$ inclusions in the $\mathrm{Fe}_{3} \mathrm{C}$ matrix is possible, their formation was hampered due to a strong interaction of the inclusions' atoms with the carbon atoms in the matrix. Our experiment driven DFT investigation uncovers the regularity in the formation of specific nitride-based NMIs depending on the type of matrix and provides important information on the possibility of the controlled formation of nitride inclusions in a particular phase during commercial steel production.

\section{Author contributions}

A. A. K.: DFT calculations, XAS analysis and writing; E. R.: contributed to the manuscript writing; H. S.: problem planning, conceptualization and writing; T. F. and M. H.: commenting on the manuscript; W. C.: calculations discussions.

\section{Conflicts of interest}

On behalf of all authors, the corresponding author states that there is no conflict of interest.

\section{Acknowledgements}

Authors acknowledge Academy of Finland grant \#311934 for the financial support. The authors acknowledge CSC - IT Center for Science, Finland for computational resources and the Centre for Material Analysis, University of Oulu for in-house characterizations. We also thank the crew of the MAX IV laboratory and Dr Ulrich Lienert, P21.2 beamline, PETRA III, Germany for their support during the beamtime operation. HS also thanks Dr Tuomas Alatarvas for providing the sample for spectro-microscopic and HEXRD measurements and Dr Frank de Groot for the discussion on the XPS data analysis.

\section{References}

1 M. Tisza and I. Czinege, Int. J. Lightweight Mater. Manuf., 2018, 1, 229-238.

2 A. M. G. Coelho and F. Bijlaard, Adv. Struct. Eng., 2010, 13, 413-429.
3 F. Javidan, A. Heidarpour, X. L. Zhao and J. Minkkinen, Thin-Walled Struct., 2016, 102, 273-285.

4 A. L. Vasconcellos da Costa e Silva, J. Mater. Res. Technol., 2018, 7, 283-299.

5 P. Ghosh and R. K. Ray, Automotive Steels, Design, Metallurgy, Processing and Applications, Elsevier, 2017, pp. 113-143.

6 M. J. Balart, C. L. Davis and M. Strangwood, Scr. Mater., 2004, 50, 371-375.

7 J. Burja, M. Kolenik, S. Zuperl and G. Klannik, Mater. Technol., 2019, 53, 919-928.

8 H. Singh, T. Alatarvas, A. A. Kistanov, S. A. Aravindh, S. Wang, L. Zhu, B. Sarpi, Y. Niu, A. Zakharov, F. M. F. de Groot, M. Huttula, W. Cao and T. Fabritius, Scr. Mater., 2021, 197, 113791.

9 F. M. F. de Groot, M. O. Figueiredo, M. J. Basto, M. Abbate, H. Petersen and J. C. Fuggle, 2p, Phys. Chem. Miner., 1992, 19, 140-147.

10 F. M. F. de Groot, J. C. Fuggle, B. T. Thole and G. A. Sawatzky, Phys. Rev. B: Condens. Matter Mater. Phys., 1990, 41, 928.

11 Y. Liang, J. Vinson, S. Pemmaraju, W. S. Drisdell, E. L. Shirley and D. Prendergast, Phys. Rev. Lett., 2017, 118, 096402.

12 J. Engel, S. Francis and A. Roldan, Phys. Chem. Chem. Phys., 2019, 21, 19011-19025.

13 Z. Peng, J. Liu, F. Huang, Q. Hu, Z. Cheng, S. Liu and Y. Cheng, Steel Res. Int., 2018, 89, 1700566.

14 R. Laskowski, Th Gallauner, P. Blaha and K. Schwarz, J. Phys.: Condens. Matter, 2009, 21, 104210.

15 G. Kresse and J. Furthmüller, Phys. Rev. B: Condens. Matter Mater. Phys., 1996, 54, 11169-11186.

16 A. Jain, S. P. Ong, G. Hautier, W. Chen, W. D. Richards, S. Dacek, S. Cholia, D. Gunter, D. Skinner, G. Ceder and K. A. Persson, APL Mater., 2013, 1, 011002.

17 J. P. Perdew, K. Burke and M. Ernzerhof, Phys. Rev. Lett., 1996, 77, 3865-3868.

18 J. K. Kesavan, D. F. Mosca, S. Sanna, F. Borgatti, G. Schuck, P. M. Tran, P. M. Woodward, V. F. Mitrović, C. Franchini and F. Boscherini, J. Phys. Chem. C, 2020, 124, 16577-16585.

19 S. Wang, A. A. Kistanov, G. King, S. Ghosh, H. Singh, S. Pallaspuro, A. Rahemtulla, M. Somani, J. Kömi, W. Cao and M. Huttula, Acta Mater., 2021, 221, 117361.

20 F. Gyakwaa, M. Aula, T. Alatarvas, T. Vuolio, Q. Shu, M. Huttula and T. Fabritius, ISIJ Int., 2020, 60, 988-997.

21 C. H. M. van Oversteeg, H. Q. Doan, F. M. F. de Groot and T. Cuk, Chem. Soc. Rev., 2017, 46, 102-125.

22 R. P. Wang, B. Liu, R. J. Green, M. U. Delgado-Jaime, M. Ghiasi, T. Schmitt, M. M. van Schooneveld and F. M. F. de Groot, J. Phys. Chem. C, 2017, 121, 24919-24928.

23 K. Lawniczak-Jablonska, T. Suski, I. Gorczyca, N. E. Christensen, K. E. Attenkofer, R. C. C. Perera, E. M. Gullikson, J. H. Underwood, D. L. Ederer and Z. L. Weber, Phys. Rev. B: Condens. Matter Mater. Phys., 2000, 61, 16623.

24 H. J. Duan, Y. Zhang, Y. Ren and L. F. Zhang, J. Iron Steel Res. Int., 2019, 26, 962-972.

25 N. Shinya, J. Kyono and K. Laha, J. Intell. Mater. Syst. Struct., 2006, 17, 1127-1133. 